\title{
Ultraviolet and Deep-Ultraviolet Excitation Photothermal Heterodyne Interferometer Combined with Semi-Micro HPLC
}

\author{
Miki IsODA, ${ }^{\dagger}$ Toshihiko ABe, Kai AOKI, and Akira HARATA \\ Department of Molecular and Material Sciences, Kyushu University, 6-1 Kasuga koen, Kasuga, Fukuoka 816-8580, \\ Japan
}

\begin{abstract}
A detection system consisting of a photothermal heterodyne interferometer (PHI) combined with semi-micro HPLC (high-performance liquid chromatography) has been designed and investigated. An ultraviolet (UV) or deep-UV laser emitting at 375 or $213 \mathrm{~nm}$, respectively, was used for the excitation of nitro-polycyclic aromatic hydrocarbons (NPAHs) and amino acids. A photothermally induced change in the refractive index of the solvent causes an optical phase difference between two arms of the interferometer, one beam passing through the photoexcited region and another used as a reference, which was sensitively detected with the PHI. The separation and detection of NPAHs and amino acids were successfully demonstrated via semi-micro HPLC with the PHI and a UV detector. The detection limits of the UVexcitation PHI for NPAHs were 1.2 - 5.2 times better than that of the commercial UV detector, although the first demonstration of deep-UV excitation suffered from significant baseline fluctuation.
\end{abstract}

Keywords Photothermal heterodyne-interferometer, semi-micro HPLC, nitro-polycyclic aromatic hydrocarbons, amino acids

(Received September 3, 2020; Accepted November 13, 2020; Advance Publication Released Online by J-STAGE November 20, 2020)

\section{Introduction}

Ultraviolet-visible (UV-VIS) absorption detection is one of the most common detection techniques in high-performance liquid chromatography (HPLC) because all molecules of practical importance absorb a part of UV-VIS light. However, the disadvantage of UV-VIS detection is its low sensitivity, especially for a small sample injection volume. The fluorescence detection reaches the single-molecule detection level, but is not tolerant toward all molecules of practical importance. When less fluorescent or nonfluorescent molecules are targets in a typical case of biological and environmental analysis, fluorescence detection requires pre- and post-column derivatization or a less-sensitive indirect detection scheme. Some pre-column derivatization reactions are time consuming, while some post-column derivatization requires additional tubing between the separation column and the detector. This extra tubing results in band broadening. Highly sensitive direct detection without derivation is ideal for HPLC analysis.

Photothermal spectroscopy based on the detection of changes in a physical property caused by heat generation via optical absorption and subsequent nonradiative relaxation is one of the most sensitive optical methods of observing nonfluorescent molecules. A photothermally induced temperature increase can be observed by probing the changes in the refractive index of a solvent with remarkable sensitivity. Tokeshi et al. reported a detection limit of 0.4 molecules of $\mathrm{Pb}(2)$ octaethylporphyrin in a $7 \mathrm{fL}$ detection volume using a thermal lens technique. ${ }^{1}$ Photothermal spectroscopy has been combined with the

† To whom correspondence should be addressed.

E-mail: satomiki@mm.kyushu-u.ac.jp separation techniques. For example, thermal lens detection is 140 - 150 times more sensitive than using a commercial UV-HPLC detector for anthraquinone dyes ${ }^{2}$ and aromatic hydrocarbons. ${ }^{3}$ Franko et al. applied the thermal lens in combination with flow injection analysis and microfluidic devices. ${ }^{4}$ Mawatari et al. succeeded in the miniaturization of separation and nonderivatized detection in a nanofluidic device for a nonfluorescent dye. ${ }^{5}$ However, it is difficult to detect less fluorescent or nonfluorescent molecules, such as biological and environmental substances, and the high versatility of photothermal spectroscopy has not been fully utilized. Therefore, our group has previously applied a deep-UV excitation thermal lens technique to the HPLC analysis of nonderivatized amino acids. ${ }^{6}$

Recently, a photothermal heterodyne interferometer (PHI) was applied to an HPLC detection system for nonderivatized environmental and biological chemical species. ${ }^{7}$ The heterodyne interferometer uses two beams, each having slightly different optical frequencies. Their recombination produces a beat signal equal to the difference in frequencies between the two beams. The beat signal is in the megahertz range, and the detector can follow it because it is many orders of magnitude lower than the frequency of the probe beam. In the PHI, the magnitude of the phase shift of the beat signals is measured instead of the divergence change or deflection of the probe beam as in thermal lens-related techniques. One arm of the interferometer passes through the photoexcited portion of the sample solution where the optical path length changes with the refraction index changes of the solvent. The phase change relative to the beam passing through another arm of the interferometer is monitored. The heterodyne interferometer has a supreme advantage in sensitively for detecting the phase shift of the beat signal, even under beamintensity fluctuation. The separation-detection performance of a 
UV-excitation PHI combined with a micro-HPLC equipped with a 6 or $18 \mathrm{~nL}$ flow cell has been reported. ${ }^{7}$

Here we report on a UV-excitation PHI combined with semimicro HPLC equipped with a $250 \mathrm{~nL}$ flow cell. A semi-micro HPLC system has high stability for daily operation, while a micro-HPLC system seems still in the making stage and less stable. The PHI detection system is optimized for a $250 \mathrm{~nL}$ flow cell, and separation and sensitive detection are demonstrated with nonderivatized chemical species having environmental and biological importance. Additionally, we demonstrate the use of a deep-UV-excitation PHI combined with semi-micro HPLC, for the first time, to enhance the versatility.

The target chemicals for UV excitation are nitro-polycyclic aromatic hydrocarbons (NPAHs) of environmental, mutagenic, and carcinogenic species. As usual for highly sensitive analysis of NPAH separation and detection with HPLC/fluorescence or chemiluminescence, NPAHs are reduced to their corresponding amino-polycyclic aromatic hydrocarbons (amino-PAHs) or reacted with chemiluminescence reagents after their reduction, respectively, ${ }^{8}$ because NPAHs exhibit only weak fluorescence and are present in low concentrations in the environment.

All of the important chemicals dissolved in liquid have optical absorption at a deep-UV wavelength at $213 \mathrm{~nm}$, whereas the acetonitrile and water of superior HPLC-eluent solvents have a little optical absorption at $213 \mathrm{~nm}$, being the shortest wavelength for the photothermal detection of dilute target solutes. Unlabeled amino acids are selected as typical target chemicals for deepUV excitation. The analysis of amino acids in biofluids is essential for biomedical diagnoses. The amino acids are generally detected after a derivatization procedure for improving the detection sensitivity. However, the derivatization of amino acids with $o$-phthalaldehyde or ninhydrin sometimes suffers from ghost peaks and baseline disturbances in the chromatogram. Although the aromatic amino acids undergo optical absorption in the UV region $(260-280 \mathrm{~nm})$, most amino acids absorb only in the deep-UV region $(200-230 \mathrm{~nm})$, based on the absorption of the carboxyl group (-COOH). ${ }^{9}$ There are several reports on UV and deep-UV detection without derivatization. ${ }^{10}$ For example, Forteschi et al. measured three aromatic amino acids in a human blood plasma using CE/deep-UV detection at $200 \mathrm{~nm}$ with the internal standard and without derivatization. ${ }^{11}$ Chung et al. developed carrier-mediated single-drop microextraction with a CE/UV detector at $200 \mathrm{~nm}$ for a 120 -fold improvement in the detection sensitivity. ${ }^{12}$ However, both methods require minimal sample manipulation. The deep-UV-excitation PHI combined with HPLC has the potential to be the best method for the separation and detection of amino acids without a derivatization process and without extra sample preparation.

\section{Experimental}

\section{Reagents and chemicals}

NPAHs and amino acids were used as received without further purification. 1-Nitronaphthalene and 9-nitroanthracene were purchased from Wako Pure Chemical Industries, Ltd., and Tokyo Chemical Industry Co., Ltd., respectively. 6-Nitrochrysene and 6-nitrobenz(a)pyrene in toluene $(100 \mu \mathrm{g} / \mathrm{mL})$ were obtained from AccuStandard, Inc. 1-Nitropyrene was obtained from Acros Organics. Amino acids (tryptophan, methionine, proline, alanine, and serine) were supplied by Kishida Chemical Co., Ltd. Water was deionized and purified using a Millipore Milli-Q system. HPLC-grade acetonitrile was purchased from SigmaAldrich. All of the solvents were filtered through membrane filters with a $0.20 \mu \mathrm{m}$ pore size, supplied by Advantec Co., Ltd.

\section{Apparatus}

The principle and the setup of the PHI combined with HPLC have been reported in detail previously, ${ }^{7}$ and thus here we only briefly describe the system by noting its modification. Two types of lasers were used as an excitation beam: a GaN diode laser (wavelength, $375 \mathrm{~nm}$; maximum power, $70 \mathrm{~mW}$; TC2003750070-45, Neoark Co.) for NPAHs and the fifth harmonics of a Nd: $\mathrm{YVO}_{4}$ laser (wavelength, $213 \mathrm{~nm}$; maximum power, $>100 \mathrm{~mW}$ at $10 \mathrm{kHz}$; pulse width, $<8 \mathrm{~ns}$; repetition frequency, $10 \mathrm{kHz}$; IMPRESS 213S, Xiton Photonics) for amino acids. Figure 1 shows schematic illustrations of the experimental setups combining semi-micro HPLC with PHI detection using $375 \mathrm{~nm}$ (a) and $213 \mathrm{~nm}$ (b) lasers. The part inside the green dashed rectangle in Fig. 1(a) is changed as shown in Fig. 1(b) for the $213 \mathrm{~nm}$ excitation. This optical system of the PHI was set on a non-air desktop active vibration isolation table (TS-140, Herz Co., Ltd.) in an acoustic enclosure (AEk-3000, Herz Co., Ltd.) to remove vibrational and environmental noises from the outside. The acoustic enclosure is indicated by the yellow dashed line in Fig. 1. The $375 \mathrm{~nm}$ laser was located inside the acoustic enclosure, and the intensity was modulated at $50 \mathrm{~Hz}$ in the $50 \%$ duty operation with a function generator (FG-273, Kenwood). The $213 \mathrm{~nm}$ laser was outside the acoustic enclosure and was used as a quasi-continuous wave modulated at $100 \mathrm{~Hz}$ with a light chopper (5844A, NF Electric Instruments). A He-Ne laser $(633 \mathrm{~nm}, 1.5 \mathrm{~mW}$, Research Electro-Optics, Inc.), whose frequency corresponds to $474 \mathrm{THz}$, was used in a frequency-stabilized operation and was divided into two beams of equal intensity, a sample beam and a reference beam, using a nonpolarizing cube half mirror (NPCH-10-6328, Sigma Koki Co., Ltd.). Both beams were passed through the acousto-optic modulators (AOMs). The AOMs shifted the frequency of the sample and reference beams by 110 and $80 \mathrm{MHz}$, respectively. The excitation beam and the sample beam were coaxially combined by a dichroic mirror and were focused on the micro flow cell connected to a semi-micro HPLC. The commercially available micro flow cell (cell volume, $250 \mathrm{~nL}$; optical pass length, $5 \mathrm{~mm}$; Cat. No. 6001-70174, GL Sciences, Inc.) for an HPLC/UV detector was used as a PHI flow cell. Its volumes are well matched to a semi-micro HPLC. Two plano-convex lenses with a focal length of $60 \mathrm{~mm}$ are used in front and back of the cell to focus the $375 \mathrm{~nm}$ excitation and probe beams in the micro flow cell and collimate them after they pass the cell. For the $213 \mathrm{~nm}$ excitation, two plano-convex lenses with a focal length of $100 \mathrm{~mm}$ are used in front and back of the cell. The lens positions are roughly optimized.

The sample beam passing through the flow cell and the reference beam were combined by a nonpolarizing cube half mirror (NPCH-10-6328, Sigma Koki Co., Ltd.). When the two beams interfered, an avalanche photodiode $(3 \mathrm{~dB}$ bandwidth; $25 \mathrm{kHz}-125 \mathrm{MHz}$; New Focus 1611-AS-FS, Newport, Ltd.) detected a beat signal at $30 \mathrm{MHz}$. The $30 \mathrm{MHz}$ beat signal was demodulated to generate an optical phase-dependent voltage, with an electrical circuits designed by Kobe Steel, Ltd. ${ }^{13}$ The output was connected to a lock-in amplifier (LI5640, NF Corporation) to measure the signal amplitude synchronized with the intensity modulation of the excitation beam at $50 \mathrm{~Hz}$ for $375 \mathrm{~nm}$ and $100 \mathrm{~Hz}$ for $213 \mathrm{~nm}$. The time constant of the lockin amplifier was set to $300 \mathrm{~ms}$.

Chromatographic separation was performed on a semi-micro HPLC system (LC-4000 series, JASCO Co.) including a pump (PU-4180HG), a column oven (CO-4060), and a UV-VIS detector (UV-4075). A reversed-phase column (InertSustain C18, $2 \mu \mathrm{m}$ particle size, $2.1 \mathrm{~mm}$ inner diameter, $150 \mathrm{~mm}$ long, GL Sciences, Inc.) was selected for NPAHs, and a hydrophilic 


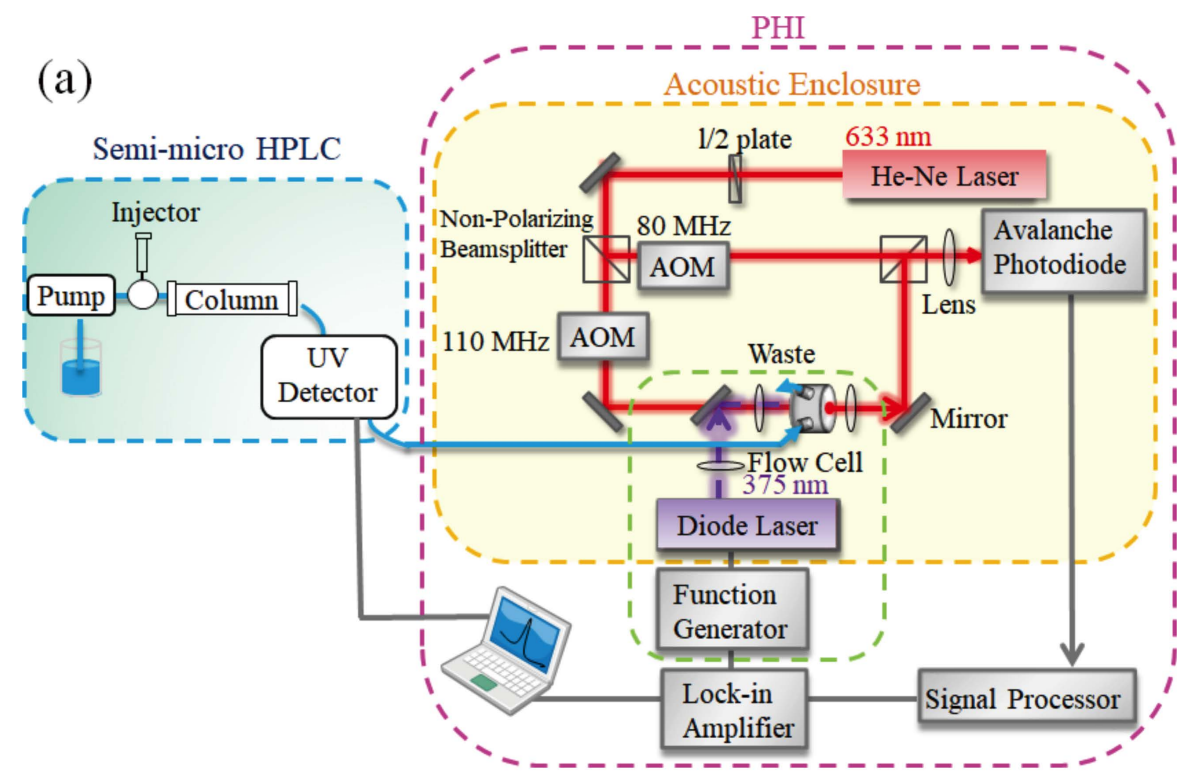

(b)

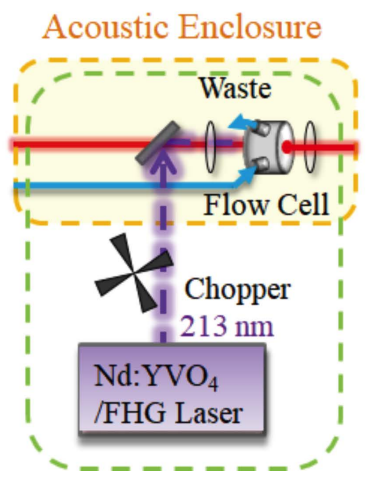

Fig. 1 Experimental apparatus of a photothermal heterodyne interferometer combined with semimicro HPLC. Excitation laser: (a) GaN diode laser $\left(375 \mathrm{~nm}\right.$ ), (b) fifth harmonic of the $\mathrm{Nd}: \mathrm{YVO}_{4}$ laser $(213 \mathrm{~nm})$.

interaction chromatography (HILIC) column (TSKgel Amide-80, $2 \mu \mathrm{m}$ particle size, $2 \mathrm{~mm}$ inner diameter, $100 \mathrm{~mm}$ long, TOSOH Corp.) was selected for amino acids. The mobile phase of both NPAHs and amino acids was $80 \%(\mathrm{v} / \mathrm{v})$ acetonitrile and $20 \%$ (v/v) water. The injection volume was $2.0 \mu \mathrm{L}$ in all of the measurements and was equal to the volume of the sample loop. Isocratic elution was performed at a flow rate of $0.2 \mathrm{~mL} / \mathrm{min}$ and a column temperature of $40^{\circ} \mathrm{C}$ for NPAHs and $20^{\circ} \mathrm{C}$ for amino acids. To compare the sensitivity, a commercial UV-VIS detector (cell volume, $12 \mu \mathrm{L}$; optical path length, $10 \mathrm{~mm}$ ) was set at $375 \mathrm{~nm}$ for NPAHs and at $213 \mathrm{~nm}$ for amino acids. The eluent from the separation column was connected directly to the flow cell for PHI detection using a fused silica capillary $(100 \mu \mathrm{m}$ inner diameter, $363 \mu \mathrm{m}$ outer diameter, TSU-100375, Polymicro Technologies) or for commercial UV detection using PEEK tubing $(130 \mu \mathrm{m}$ inner diameter, 1/16" outer diameter, 601037305 , GL Sciences). The UV detector values and the amount of output voltage of the lock-in amplifier in the PHI detection were sent to a computer using the Jasco LC-NET II/ADC2. Chromatograms were acquired at a data-sampling frequency of $2 \mathrm{~Hz}$ and analyzed using ChromNAV 2.0 HPLC software. All measurements were conducted under steady-state conditions at room temperature $\left(25 \pm 2^{\circ} \mathrm{C}\right)$.

\section{Results and Discussion}

The newly developed instrument of semi-micro HPLC separation and UV-excitation PHI detection was optimized with 1-nitropyrene $1 \times 10^{-5} \mathrm{M}$ in an 80:20 (v/v) acetonitrile-water mixture as a sample. For superior PHI chromatograms (chromatograms measured with PHI detection) to detect separation, a variety of factors related to the signal-tobackground ratio of the PHI were examined: the focal lengths and the positions of lenses placed in front of and at the back of the flow cell, for focusing both the excitation and probe beams; the modulation frequency of the excitation beam; and the flow rate and various conditions of separation.

The optimal flow rate depends on the column dimensions. Typically, a conventional analytical column with a $4.6 \mathrm{~mm}$ internal diameter is used with a $1.0 \mathrm{~mL} / \mathrm{min}$ flow rate. The same linear velocity is achieved with a $2 \mathrm{~mm}$ inner diameter column using a flow rate of $0.20 \mathrm{~mL} / \mathrm{min}$ as long as there are no leaks. Figure 2 shows the dependence of the background fluctuation of PHI chromatograms on the flow rate at 0.15 $0.30 \mathrm{~mL} / \mathrm{min}$. The baseline at 0.25 and $0.30 \mathrm{~mL} / \mathrm{min}$ showed a large fluctuation. The optimal flow rate was found to be 


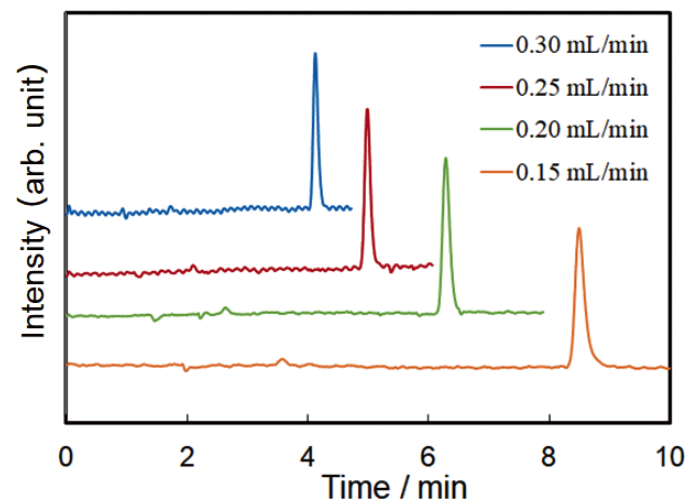

Fig. 2 Chromatograms of 1-nitropyrene $\left(10^{-5} \mathrm{M}\right)$ at different flow rates via PHI detection. Excitation laser, GaN diode laser $(375 \mathrm{~nm})$ column, InertSustain C18; mobile phase, 80:20 (v/v) acetonitrilewater.

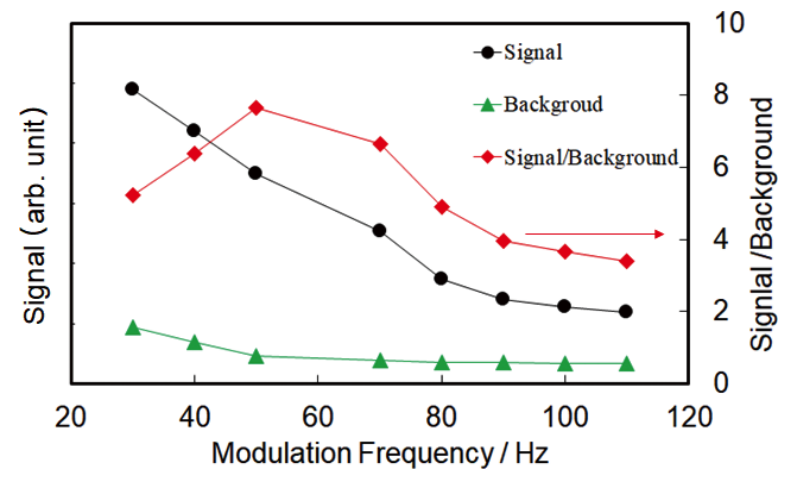

Fig. 3 Modulation frequency dependence of the PHI signal (•), background signal $(\boldsymbol{\Delta})$, and signal to background $(\bullet)$. Excitation laser, $\mathrm{GaN}$ diode laser $(375 \mathrm{~nm}) ; 1 \times 10^{-5} \mathrm{M}$ 1-nitropyrene in 80:20 (v/v) acetonitrile-water; flow rate, $0.2 \mathrm{~mL} / \mathrm{min}$.

$0.20 \mathrm{~mL} / \mathrm{min}$, where both the baseline fluctuation and the width of the signal peak were small. This means that the entire analytical system minimized the extra dead volume. Although a thermal lens is not suitable for gradient separation, ${ }^{6,14}$ minimization of the extra dead volume is especially critical for application to future gradient separation. At the flow rate, the dependence of the signal-to-background ratio of the PHI on a modulation frequency of between 30 and $110 \mathrm{~Hz}$ was investigated, as shown in Fig. 3. Note that data at a power source frequency of $60 \mathrm{~Hz}$ were not measured. The magnitudes of the signal and background signal were evaluated by measuring the peak intensity and the average of the signal intensity of each chromatogram during the initial $6 \mathrm{~min}$, respectively. The background signal is partly caused by photo-absorption of the pure solvent, which is modulation-frequency dependent, along with some electronic sources of unconfirmed frequency dependences. It is natural that the signal and background magnitudes decrease with the increasing modulation frequency, because a temperature increase causes the PHI signal, and the accumulation time of photo-induced heat dominates the magnitudes against thermal diffusion and flow-induced heat sweep-out. ${ }^{15}$ The signal-to-background ratio has a peak at $50 \mathrm{~Hz}$. At a frequency lower than $50 \mathrm{~Hz}$, the background signal showed a stronger frequency dependence than the signal. The

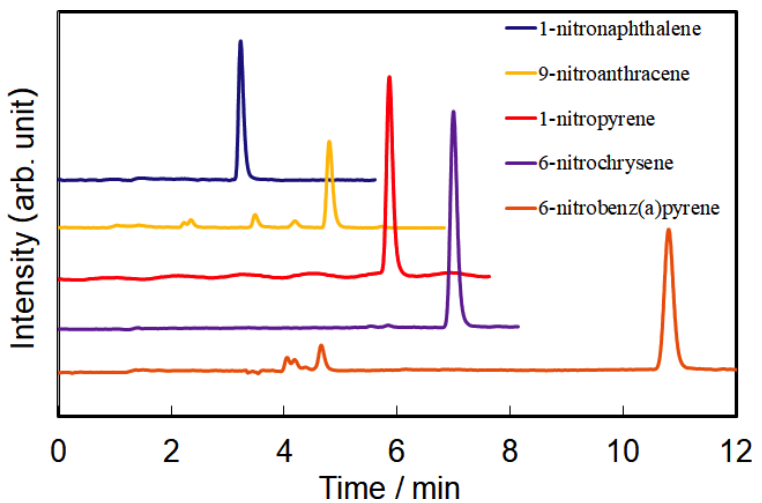

Fig. 4 Chromatograms of single-component NPAHs via PHI detection. All concentrations were $1.0 \times 10^{-5} \mathrm{M}$. Excitation laser, $\mathrm{GaN}$ diode laser (375 $\mathrm{nm}$ ); column, InertSustain $\mathrm{C} 18$; mobile phase, 80:20 (v/v) acetonitrile-water; flow rate, $0.2 \mathrm{~mL} / \mathrm{min}$.

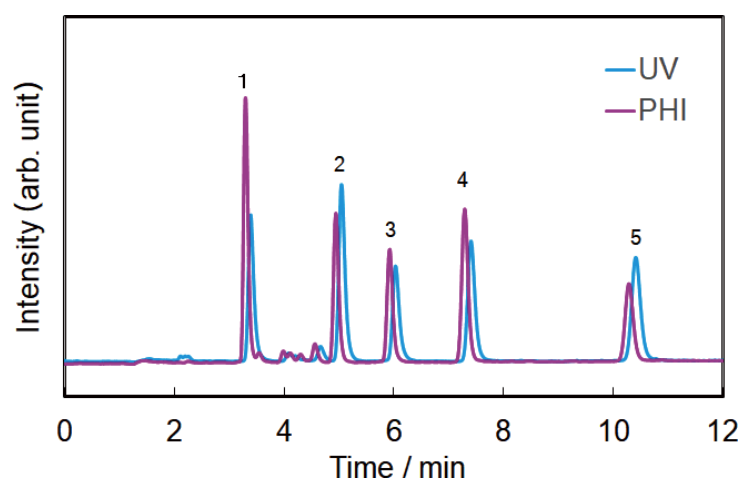

Fig. 5 Chromatogram of a mixture of five NPAHs via UV and PHI detection. Peaks: (1) 1-nitronaphthalene, $1.2 \times 10^{-5} \mathrm{M}$; (2) 9-nitroanthracene, $1.2 \times 10^{-5} \mathrm{M}$; (3) 1-nitropyrene, $2.4 \times 10^{-6} \mathrm{M}$; (4) 6-nitrochrysene, $1.2 \times 10^{-6} \mathrm{M}$; (5) 6-nitrobenz(a)pyrene, $1.2 \times 10^{-6} \mathrm{M}$. Excitation laser of PHI, GaN diode laser $(375 \mathrm{~nm})$; column, InertSustain C18; mobile phase, 80:20 (v/v) acetonitrile-water; flow rate, $0.2 \mathrm{~mL} / \mathrm{min}$.

modulation frequency of $50 \mathrm{~Hz}$ was selected as an optimized condition. All UV-excitation experiments were performed using the optimized optical configuration, optimal flow rate $(0.20 \mathrm{~mL} /$ $\min )$, and optimal modulation frequency $(50 \mathrm{~Hz})$.

Figure 4 shows reversed-phase chromatograms of a pure component using a PHI detector. The chromatograms of 1-nitronaphthalene, 1-nitropyrene, and 6-nitrochrysene contained only one peak. Meanwhile, unknown impurities were found in 9-nitroanthracene and 6-nitrobenz(a)pyrene chromatograms. Figure 5 shows a reversed-phase chromatogram of a mixture of five NPAHs using a PHI and a UV detector. Five kinds of NPAHs have been separated successfully and analyzed with a PHI and a commercial UV detector. Each peak is well-assigned to each component, including peaks of the unknown impurities. The retention time, $t_{\mathrm{R}}$, and effective number of theoretical plates, as well as the half width (full width at half maximum, $W_{1 / 2}$ ), are summarized in Table 1. The difference in the retention times for each sample between the PHI and UV detector was $0.1 \mathrm{~min}$. This time delay corresponds to the difference in the distance of the PHI or UV detector from the separation column. The effective number of theoretical plates is calculated as to be 5.54 
Table 1 Comparison of the chromatographic parameters and detection limits $(3 \sigma)$ for NPAHs using UV and PHI detection

\begin{tabular}{|c|c|c|c|c|c|c|c|c|c|}
\hline \multirow[b]{2}{*}{ Analyte } & \multirow[b]{2}{*}{$\begin{array}{c}\text { Molar } \\
\text { absorptivity/ } \\
\mathrm{cm}^{-1} \mathrm{M}^{-1}\end{array}$} & \multicolumn{4}{|c|}{ UV } & \multicolumn{4}{|c|}{ PHI } \\
\hline & & $\begin{array}{l}\text { Retention } \\
\text { time/min }\end{array}$ & $\begin{array}{l}\text { Effective } \\
\text { number of } \\
\text { theoretical } \\
\text { plates (-) }\end{array}$ & $\begin{array}{l}\text { Half } \\
\text { width/ } \\
\text { min }\end{array}$ & $\begin{array}{l}\text { Detection } \\
\text { limit/nM }\end{array}$ & $\begin{array}{l}\text { Retention } \\
\text { time/min }\end{array}$ & $\begin{array}{l}\text { Effective } \\
\text { number of } \\
\text { theoretical } \\
\text { plates (-) }\end{array}$ & $\begin{array}{l}\text { Half } \\
\text { width/ } \\
\text { min }\end{array}$ & $\begin{array}{l}\text { Detection } \\
\text { limit/nM }\end{array}$ \\
\hline 1-Nitronaphthalene & 2200 & 3.4 & 5300 & 0.11 & 53 & 3.3 & 7500 & 0.09 & 31 \\
\hline 9-Ninroantthacene & 3000 & 5.0 & 9600 & 0.12 & 25 & 4.9 & 13000 & 0.10 & 14 \\
\hline 1-Nitropyrene & 12000 & 6.0 & 12000 & 0.13 & 15 & 5.9 & 16000 & 0.11 & 2.9 \\
\hline 6-Nitrochysene & 9900 & 7.4 & 15000 & 0.14 & 41 & 7.3 & 17000 & 0.13 & 27 \\
\hline 6-Nitrobenz(a)pyrene & 13000 & 10.4 & 21000 & 0.17 & 34 & 10.3 & 23000 & 0.16 & 28 \\
\hline
\end{tabular}

$\left(t_{\mathrm{R}} / W_{1 / 2}\right)^{2}$ for each peak; it thus represents not only the HPLC column performance, but also the overall performance of the separation-detection system, as described below. The number of theoretical plates is primarily defined for measuring the HPLC column performance, and the columns with a high number of theoretical plates are considered to be more efficient. Although the same column was used both for PHI and UV detection, the effective numbers of theoretical plates for the PHI were higher than those for UV detection. The factors causing a low number of theoretical plates include the dead volumes of the HPLC systems. Apparently, the smaller flow-cell volume of the PHI results in less chromatographic broadening.

The HPLC calibration curves for the peak height of the chromatogram of the NPAHs, according to the UV and PHI detectors, are shown in Fig. S1 (Supporting Information). All data in Fig. S1 represent the averaged values from three measurements. The concentration ranges examined were $10^{-8}-10^{-5} \mathrm{M}$ for 1-nitropyrene with the PHI and $10^{-7}-10^{-5} \mathrm{M}$ for the others. The signal intensity of the NPAHs was linear, with a concentration of over three or four orders of magnitude. Thus, the linear dynamic range of the PHI could be almost the same as that of UV.

The UV and PHI detection limits are examined as the values of three times the standard deviation $(3 \sigma)$ of the blank. The blank signal is analogous to the chromatographic baseline. ${ }^{16}$ The standard deviation $(\sigma)$ of the blank signal was estimated from half of the short-term peak-to-peak noise in $1.0-1.8 \mathrm{~min}$, corresponding to ten-times the range of the peak half width on a chromatogram. The detection limits $(3 \sigma)$ determined for NPAHs are summarized in Table 1, together with the molar absorptivity, retention time, effective number of theoretical plates, and half width at half maximum. The detection limits of NPAHs with the PHI are $1.2-5.2$ times better than those with the conventional UV detector. The concentration detection limit for 1-nitropyrene in this work was better than that in the previous work (24 nM) with a PHI/micro HPLC system, ${ }^{7}$ even when compared with the detection limit of the injected sample; the detection limit was $24 \mathrm{fmol}$ in the previous work and is $5.8 \mathrm{fmol}$ in this paper. Though the small-diameter column $(1 \mathrm{~mm}$ inner diameter) used in the PHI/micro HPLC system can improve the separation performance by reducing the on-column sample dilution, a larger mismatch in volumes between $1.0 \mu \mathrm{L}$ of the sample injection and 6 or $18 \mathrm{~nL}$ of the flow cells caused a poorer concentration sensitivity accompanied by band broadening. In the PHI/semi-micro HPLC system in this study, the use of a $250 \mathrm{~nL}$ flow cell relaxed the mismatch, although the separation performance with the $2.1 \mathrm{~mm}$ inner diameter column might not be the best. As shown in Table 1, the NPAH peaks become broader at a longer retention time because of diffusion.

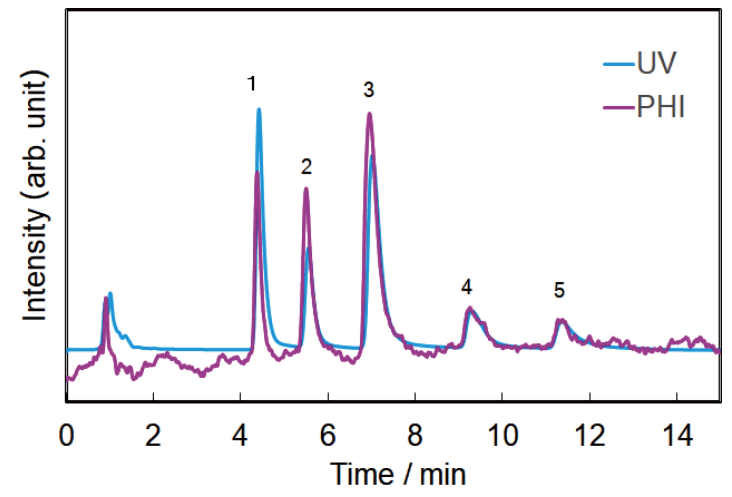

Fig. 6 Chromatograms of a mixture of five amino acids via UV(detection wavelength: $213 \mathrm{~nm}$ ) and deep-UV-excitation PHI detection. Peaks: (1) L-tryptophan, $20 \mu \mathrm{M}$; (2) L-methionine, $0.2 \mathrm{mM}$; (3) L-proline, $10 \mathrm{mM}$; (4) L-alanine, $2 \mathrm{mM}$; (5) L-serine, $2 \mathrm{mM}$. Excitation laser of the PHI, fifth harmonics of the Nd:YVO laser $(213 \mathrm{~nm})$; column, TSKgel Amide-80; mobile phase, 80:20 (v/v) acetonitrilewater; flow rate, $0.2 \mathrm{~mL} / \mathrm{min}$

There is still room for separation-performance improvements in the PHI/semi-micro HPLC system; however, it was apparent that the system showed better stability than the $\mathrm{PHI} / \mathrm{micro}$ HPLC system both in the long term and in the short term, seen as a stable baseline in chromatograms.

The developed PHI/semi-micro HPLC system is applicable to a wide range of excitation wavelengths if appropriate optical parts transport and focus the excitation beam to the flow cell. The first demonstration of deep-UV excitation at $213 \mathrm{~nm}$ and PHI detection combined with semi-micro HPLC separation is shown in Fig. 6. All samples could be eluted in almost the same time by adjusting the distance of the flow cell from the separation column for the commercial UV and PHI detectors. A mixture of five unlabeled amino acids was separated successfully with the HILIC phase chromatogram. Although individual calibration plots were not measured for both the UV and PHI detectors, the detection limits of each amino acid were estimated from the signal-to-noise ratio. The detection limits of methionine were $2 \mu \mathrm{M}$ for UV and $20 \mu \mathrm{M}$ for the PHI, while those of serine were $70 \mu \mathrm{M}$ for UV and $1 \mathrm{mM}$ for the PHI. The PHI detection limits of five amino acids were 10-23 times higher than the UV detection limits. The chromatogram with the PHI detector suffered from an instable baseline as compared with that with the commercial UV detector, even after optimization of the position and focal length of the lenses for the excitation beam and of the chopping frequency. First, laser- 
intensity instability can cause baseline fluctuation in the PHI detection through the inherent optical absorption of pure solvents with weak, but certain, strength. The $213 \mathrm{~nm}$ laser can operate with high stability, with an intensity fluctuation of less than $1 \%$ per hour, although its use as a quasi-continuous wave can be a contributing factor in fluctuation. One of the most plausible factors in fluctuation is impurities in acrylonitrile. Commercially available HPLC-grade acetonitrile contains acrylonitrile, even at concentrations on the order of $10^{-5} \mathrm{M} \cdot{ }^{17}$ Micro-inhomogeneity in a mixture of water and acrylonitrile may help the fluctuation. ${ }^{18}$ The one-photon or two-photon excitation can be caused by the impurities and some contaminations in the solvents, as well as by optical elements inside the flow cell under the high photon density of a $213 \mathrm{~nm}$ laser. Since commercially available highly pure solvents for HPLC with fluorescence detection enhance the sensitivity as is, it is desirable for extremely pure acetonitrile for HPLC to be commercially available. Otherwise, the additional optical setup for PHI detection should be modified to achieve high sensitivity and good versatility simultaneously. Photothermal signal enhancement by the transient absorption of photoexcited states is one good candidate for an additional setup. ${ }^{19,20}$

\section{Conclusions}

An ultraviolet- and deep-ultraviolet-excitation photothermal heterodyne interferometer (PHI) combined with a semi-micro high-performance liquid chromatograph has been developed. Mixtures of nitro-polycyclic aromatic hydrocarbons or amino acids were successfully separated and detected. The sensitivity of the PHI was 1.2 - 5.2 times better than that of the commercial UV detector for $375 \mathrm{~nm}$ excitation. As for the $213 \mathrm{~nm}$ excitation, unlabeled amino acids, including proline, alanine, and serine, were separated and detected with the PHI, although the chromatogram suffered from an instable baseline as compared with that with the commercial UV detector, suggesting the necessity of improving the purity of solvents as well as the optical configuration of the PHI detection system. The system of ultraviolet- and deep-ultraviolet-excitation HPLC/PHI is expected to become a powerful tool for the direct analysis of environmental and biorelated substances.

\section{Acknowledgements}

A method for heterodyne-interferometric detection of a photothermal signal developed by Kobe Steel, Ltd., was used. This work was supported by a Grant-in-Aid for Scientific Research (KAKENHI) [Nos. JP26288068 and JP18H03915] from the Japan Society for the Promotion of Science.

\section{Supporting Information}

This material is available free of charge on the Web at http:// www.jsac.or.jp/analsci/. Some descriptions of the principle and advantages of the PHI are given. The way to access Ref. 13 is noted as well.

\section{References}

1. M. Tokeshi, M. Uchida, A. Hibara, T. Sawada, and T. Kitamori, Anal. Chem., 2001, 73, 2112.

2. F. Li, A. A. Kachanov, and R. N. Zare, Anal. Chem., 2007, 79, 5264.

3. S. Hiki, K. Mawatari, A. Hibara, M. Tokeshi, and T. Kitamori, Anal. Chem., 2006, 78, 2859.

4. M. Franko, M. Liu, A. Boškin, A. Delneri, and M. A. Proskurnin, Anal. Sci., 2016, 32, 23.

5. Y. Tsuyama, K. Morikawa, and K. Mawatari, J. Chromatogr. A, 2020, 1624, 461265.

6. H. Katae, S. Hirashima, and A. Harata, J. Phys., 2010, 214, 012122 .

7. M. Isoda, M. Fukuma, and A. Harata, Anal. Sci. 2019, 35, 1311.

8. K. Hayakawa, Chem. Pharm. Bull., 2016, 64, 83.

9. D. B. Wetlaufer, Adv. Protein Chem., 1963, 17, 303.

10. Y. Song, C. Xu, H. Kuroki, Y. Liao, and M. Tsunoda, J. Pharm. Biomed. Anal., 2018, 147, 35.

11. M. Forteschi, S. Sotgia, S. Assaretti, D. Arru, D. Cambedda, E. Sotgiu, A. Zinellu, and C. Carru, J. Sep. Sci., 2015, 38, 1794.

12. J. Choi, K. Choi, J. Kim, A. Y. B. H. Ahmed, Z. A. AlOthman, and D. S. Chung, J. Chromatogr. A, 2011, 1218, 7227.

13. E. Takahashi, R. Katayama, and H. Takamatsu, EP Patent, 2008, 1925928.

14. J. K. Logar, A. Malej, and M. Franko, Rev. Sci. Instrum., 2003, 74, 776.

15. S. Hirashima, H. Katae, and A. Harata, Jpn. J. Appl. Phys., 2009, 48, 07GE04-1.

16. J. P. Foley and J. D. Dorsey, Chromatographia, 1984, 18, 503.

17. K. Sano and T. Ito, JP Patent, 2016, 6038157.

18. T. Takamuku, M. Tabata, A. Yamaguchi, J. Nishimoto, M. Kumamoto, H. Wakita, and T. Yamaguchi, J. Phys. Chem. $B, \mathbf{1 9 9 8}, 102,8880$.

19. A. Harata, K. Fukushima, and Y. Hatano, Anal. Sci., 2002, $18,1367$.

20. S. Hirashima and A. Harata, Jpn. J. Appl. Phys., 2008, 47, 3970. 